\title{
Visual estimation versus software fusion for MRI-targeted biopsy
}

Although the use of MRI-ultrasonography fusion is more histologically informative than visual estimation for MRI-targeted biopsy, a recent prospective blinded study published in European Urology has shown that rates of cancer detection are similar for the two approaches.

In-gantry prostate biopsy of MRIsuspicious regions using real-time magnetic resonance guidance is the gold standard approach to targeted biopsy, but this technique is only available at

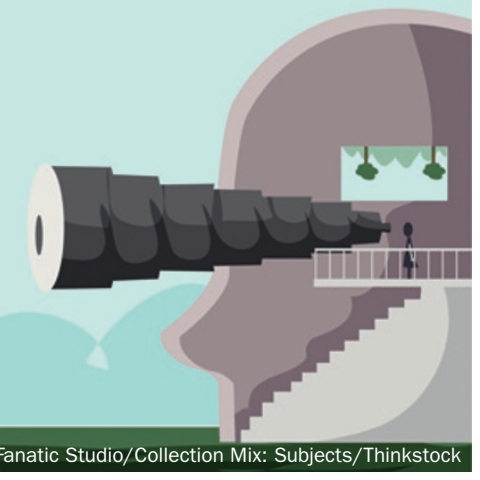

a few centres and is limited by several challenges, including a steep learning curve. In its place, investigators use either visual estimation (whereby the surgeon samples a visually estimated area on ultrasonography that corresponds to the suspicious region on MRI) or software fusion of prebiopsy MRI scans with real-time ultrasonography images.

In this latest study of 145 men (with 172 MRI targets), two MRI-ultrasonographyfusion-targeted cores per target were sampled by a single operator, before a second operator took samples from two visually targeted cores per target and a standard 12-core biopsy. Fusion biopsy detected 55 cancers in total (including 35 Gleason sum $\geq 7$ cancers) compared with 46 cancers (including 26 Gleason sum $\geq 7$ cancers) using visual estimation.

This difference was not statistically significant, but Wysock et al. observed a trend towards increased detection with fusion biopsy for all study subsets (particularly for men with smaller lesions), suggesting that larger studies are needed before a benefit of MRI-ultrasonography fusion targeted biopsy can be ruled out. Furthermore, fusion biopsy provided nonbenign histological information for 77 targets compared with 60 targets using visual targeting, highlighting a clear advantage of using this technique.

Although the cancer detection rate was greater with standard biopsy than with targeted biopsy, the latter identified all cancers with Gleason sum $\geq 7$. Overall, more cancer was found on a per-lesion and per-core basis with targeted biopsy; until more data are available, the authors recommend targeted biopsy with software fusion for men with smaller tumours.

Melanie Clyne

Original article Wysock, J. S. et al. A prospective, blinded comparison of magnetic resonance (MR) imagingultrasound fusion and visual estimation in the performance of MR-targeted prostate biopsy: the PROFUS trial. Eur. Urol. doi:10.1016/j.eururo.2013.10.048 